\title{
Response of Recurrent High-Grade Glioma to Treatment with ${ }^{90}$ Y-DOTATOC
}

Dirk Heute ${ }^{1}$, Herwig Kostron ${ }^{2}$, Elisabeth von Guggenberg ${ }^{1}$, Shota Ingorokva ${ }^{2}$, Michael Gabriel $^{1}$, Georg Dobrozemsky ${ }^{1}$, Günther Stockhammer ${ }^{3}$, and Irene J. Virgolini ${ }^{1}$

${ }^{I}$ Department of Nuclear Medicine, Medical University of Innsbruck, Innsbruck, Austria; ${ }^{2}$ Department of Neurosurgery, Medical University of Innsbruck, Innsbruck, Austria; and ${ }^{3}$ Department of Neurology, Medical University of Innsbruck, Innsbruck, Austria

The treatment of patients with high-grade malignant glioma still represents an unsolved clinical problem. We report the treatment of 3 patients who had World Health Organization grade IV recurrent glioblastoma: a 23-y-old woman and 2 men aged 61 and $62 \mathrm{y}$. Methods: All 3 patients were treated with the somatostatin receptor radiopharmaceutical ${ }^{90}$ Y-labeled $[1,4,7,10$-tetraazacyclododecane- $N, N^{\prime}, N^{\prime \prime}, N^{\prime \prime \prime}$-tetraacetic acid ${ }^{0}$-D-Phe $\left.{ }^{1}, \mathrm{Tyr}^{3}\right]$ octreotide (DOTATOC). A cumulated dose of 1.7-2.2 GBq given in 3 or 4 cycles was locally injected into a previously implanted catheter system. Results: Treatment was successful in all 3 patients, with only minor side effects reported. After treatment, MRI and PET showed complete remission in one patient and partial remission in the other patients. These findings correlated well with clinical improvement and improved quality of life. Conclusion: Receptormediated radionuclide therapy by locally injected ${ }^{90} \mathrm{Y}$-DOTATOC is feasible and well tolerated. This approach represents an attractive strategy for the treatment of locally recurring or progressing glioblastoma.

Key Words: glioblastoma; intracavitary treatment; ${ }^{90}$ Y-DOTATOC

J Nucl Med 2010; 51:397-400

DOI: 10.2967/jnumed.109.072819

$\mathbf{T}$ he survival rate of patients with glioblastoma has changed little over the past 30 years (1). A minor increase in survival from 12.1 to $14.6 \mathrm{mo}$ was achieved by the addition of temozolomide to standard radiotherapy (2). Furthermore, attempts with local chemotherapy have shown some benefit and have been introduced into clinical routine (3). However, no substantial improvement in prognosis has been achieved so far. Regulatory peptide receptors such as the vasoactive intestinal peptide or the somatostatin receptor are known to be overexpressed in numerous human cancers (4). These receptors have been used as molecular targets by which radiolabeled peptides can localize tumor cells in vivo $(5,6)$ and, more recently, to treat cancers such as malignant gliomas (7) by peptide receptor-mediated radionuclide therapy $(8)$. We have previously shown that

Received Mar. 5, 2009; revision accepted Dec. 8, 2009.

For correspondence or reprints contact: Irene J. Virgolini, Department of Nuclear Medicine, Medical University of Innsbruck, Anichstrasse 35, A-6020 Innsbruck, Austria.

E-mail: irene.virgolini@i-med.ac.at

COPYRIGHT @ 2010 by the Society of Nuclear Medicine, Inc. intratumoral treatment with ${ }^{90}$ Y-labeled 1,4,7,10-tetraazacyclododecane- $N, N^{\prime}, N^{\prime \prime}, N^{\prime \prime \prime}$-tetraacetic acid (DOTA) lanreotide presents an alternative approach to systemic application of the radioligand (9). This method has proven safe and yields a higher tumor dose with less radiation exposure for critical organs and the whole body. Patients with glioblastoma reported an improved quality of life (9). Recently, local treatment with the somatostatin receptor analog octreotide has been proposed for patients with recurrent medulloblastoma or glioma of World Health Organization (WHO) grade II or III $(10,11)$. The aim of this study was to prove the feasibility and safety of locally injected ${ }^{90}$ Y-labeled [1,4,7,10-tetraazacyclododecane- $N, N^{\prime}, N^{\prime \prime}, N^{\prime \prime \prime}$-tetraacetic acid $^{0}$-D-Phe ${ }^{1}$, $\mathrm{Tyr}^{3}$ ]octreotide (DOTATOC) and to evaluate the clinical impact of this therapy on a small group of patients with grade IV glioblastoma.

\section{MATERIALS AND METHODS}

We report the treatment of 3 patients with histologically proven glioblastoma multiforme (WHO grade IV): patient 1, a 24-y-old woman; patient 2, a 61-y-old man; and patient 3, a 62-y-old man. The patients had undergone maximal cytoreductive surgery and had received radiochemotherapy with temozolomide (1 cycle with $200 \mathrm{mg} / \mathrm{m}^{2}$ over $5 \mathrm{~d}$ ) followed by external-beam radiotherapy with a tumor boost (60 Gy in the tumor region) and concomitant temozolomide therapy $\left(75 \mathrm{mg} / \mathrm{m}^{2}\right)$. Despite this treatment, followup MRI studies revealed tumor recurrence, with peritumoral edema and garland-shaped and nodular contrast enhancement surrounding the surgical cavity (Fig. 1A). In accordance with this finding, ${ }^{18} \mathrm{~F}$-FDG PET showed increased glucose metabolism adjacent to the tumor cavity (Fig. 1C). Therefore, radiationinduced tumor pseudoprogression could be excluded. At the same time, somatostatin receptor PET was performed after intravenous injection of $100 \mathrm{MBq}$ of ${ }^{68} \mathrm{Ga}$-labeled DOTATOC (6). On the basis of these findings, local radionuclide therapy with ${ }^{90}$ Y-DOTATOC was considered. A subcutaneous reservoir system (Rickham Reservoir; Codman Shurtleff Inc.) was implanted into the resection cavity for local application of the radiopharmaceutical (12).

In all 3 patients, individual dosimetric calculations were performed on the basis of repeated whole-body images taken $30 \mathrm{~min}$ to $72 \mathrm{~h}$ after local injection of $80 \mathrm{MBq}$ of ${ }^{111} \mathrm{In}$-labeled DOTATOC into the Rickham reservoir (Figs. 2A-2D). Regions of interest were drawn around the critical organs and the tumor cavity 
FIGURE 1. Response of high-grade glioma to local therapy with cumulated 2.2-GBq dose of ${ }^{90} \mathrm{Y}$-DOTATOC, given in 3 cycles (from left to right: study before therapy, control study 3 mo after second dose, control study 3 mo after third dose, and control study 23 mo after third dose). (A) T1-weighted enhanced MR images show diminishing contrast agent in tissue surrounding resection cavity throughout therapy. (B) ${ }^{68} \mathrm{Ga}-\mathrm{DOTATOC}$ PET images representing somatostatin receptor status show increased tracer uptake around resection cavity before therapy and normalization in control studies. (C) ${ }^{18} \mathrm{~F}-\mathrm{FDG}$ PET images show highly increased glucose metabolism at rim of tumor cavity before therapy and diminishing uptake after therapy. (D) T1weighted unenhanced MR image before therapy shows previously implanted reservoir system well positioned in resection cavity in left parietal lobe. Follow-up images ( ${ }^{18} \mathrm{~F}$-fluoroethyltyrosine PET) show that ratio of uptake per pixel was lower in region adjacent to tumor cavity than in contralateral region, giving no evidence of residual or relapsing tumor.

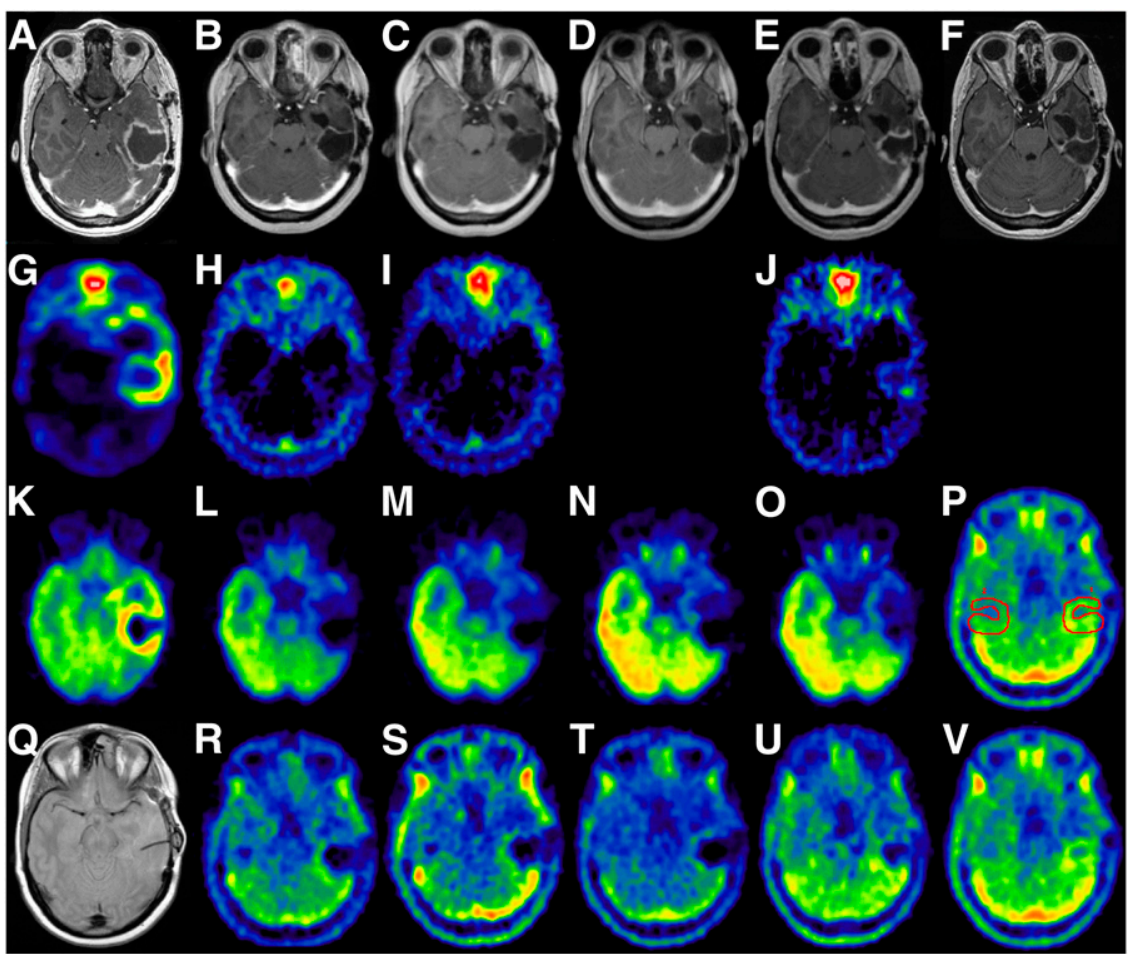

(Fig. 2C). These images helped to exclude leakage of the reservoir into the cerebral fluid.

At the time of admission, the patients showed a Karnofsky performance score of $60 \%-70 \%$ and neurologic symptoms such as hemiplegia, headache, nausea, vomiting, and blurred vision. Beyond these, an organic psychosyndrome that included difficulties in word finding and speech comprehension was found.

In view of the presence of advanced, progressive tumor, no standard therapeutic option was left. A surrogate therapy consisting of a locally injected radiolabeled compound was agreed upon by an interinstitutional tumor board consisting of neurosurgeons, neuroradiologists, radiotherapists, and nuclear medicine physicians. The patients gave informed consent to undergo the surrogate therapy.

Starting 1-2 mo after the diagnosis of tumor recurrence, 1,660$2,220 \mathrm{MBq}$ of ${ }^{90}$ Y-DOTATOC were given in 3 or 4 fractions at an interval of 3-4 mo (Table 1). The dose was chosen on the basis of studies by Ferrari et al. (13), and the 3-mo gap between therapeutic fractions was allowed so that we could check for possible side effects. Each fraction was prepared with $4 \mathrm{~mL}$ of saline and injected slowly, over $10 \mathrm{~min}$, into the previously implanted Rickham reservoir.

During the entire treatment period, the patients continued taking their current anticonvulsive medication (carbamazepine, $800 \mathrm{mg} / \mathrm{d}$ ) or antiedemic medication (dexamethasone, $24 \mathrm{mg} / \mathrm{d}$, gradually reduced to $4 \mathrm{mg} / \mathrm{d}$ ).

Follow-up imaging was performed using contrast-enhanced MRI and ${ }^{68} \mathrm{Ga}$-DOTATOC, ${ }^{18} \mathrm{~F}$-FDG, and ${ }^{18} \mathrm{~F}$-fluoroethyltyrosine PET.

\section{RESULTS}

The ${ }^{68} \mathrm{Ga}$-DOTATOC PET scans showed increased activity indicating expression of somatostatin receptor 2 . Depending on the distribution of recurrent tumor tissue, a characteristic uptake pattern was seen. Especially in patient 1, a distinct circle of activity was seen surrounding the surgical cavity (Fig. 1).

Scintigraphic images after local injection of ${ }^{111} \mathrm{In}$ DOTATOC proved that the radiolabeled compound was well localized within the surgical resection cavity and the reservoir system (Fig. 2). For $2 \mathrm{~d}$ after injection of the tracer, only a slight accumulation of radioactivity could be found in the kidneys and liver. Based on individual dosimetric calculations, the mean dose of ${ }^{90}$ Y-DOTATOC was $98 \mathrm{~Gy} / \mathrm{GBq}$ to residual tumor tissue surrounding the tumor cavity and $2.8 \mathrm{~Gy} / \mathrm{GBq}$ to the kidneys. The mean effective dose to the whole body was $0.56 \mathrm{~Sv} / \mathrm{GBq}$.

The only adverse effects observed were recurrence of an epileptic seizure in one patient and a transient, mild headache on the day of radiopharmaceutical application in another patient. In neither case was any special treatment necessary.

There was a complete remission in one of the patients and partial remission in the other two. After treatment, the Karnofsky performance score increased, by $10 \%-40 \%$, in all 3 patients (Table 2). All patients reported an improved quality of life and a stabilization of their mood. Neurologic 


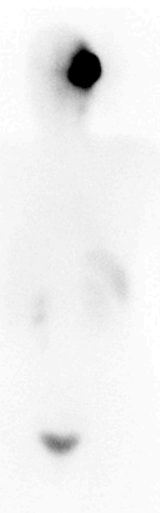

$\mathbf{E}$

B
C

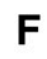

D
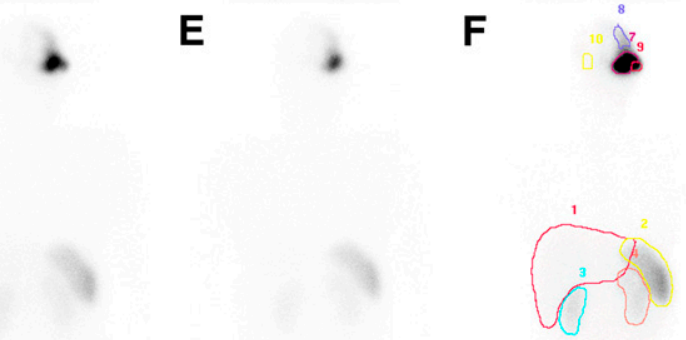

FIGURE 2. Whole-body $\gamma$-camera images of same patient as in Figure 1 after injection of $80 \mathrm{MBq}$ of ${ }^{111} \mathrm{In}$-labeled DOTATOC into Rickham reservoir: conjugated view 30 min after injection (A); conjugated view $4 \mathrm{~h}$ after injection (B); conjugated view $24 \mathrm{~h}$ after injection, including regions of interest used for dosimetric calculations (C); and conjugated view $72 \mathrm{~h}$ after injection (D). Images represent geometric mean of anterior and posterior views, have been set to same absolute window level to emphasize decay, and build basis for individual dosimetric calculations. Lack of tracer is seen in ventricular system and spinal canal, and only minor activity is transported from treatment region to rest of body.

and neuropsychologic symptoms such as paralysis, difficulties in word finding, and difficulties in speech comprehension gradually decreased. Two patients could even return to work. Four years later, patient 1 is still alive and is showing a constant Karnofsky performance score of
$100 \%$, whereas the other 2 patients died of tumor progression 10-13 mo after admission to the study.

\section{MRI Evaluation}

In one patient, the follow-up studies showed a steady decrease in contrast agent surrounding the tumor cavity. In the other 2 patients, MRI showed signs of necrosis, such as central hypoperfusion and stripes in the distribution of contrast agent, along with an increase in contrast medium outside the therapeutic target volume.

\section{PET Evaluation}

The maximum standardized uptake value for ${ }^{18} \mathrm{~F}-\mathrm{FDG}$ in the target region adjacent to the tumor cavity decreased in patients 1 and 2-from 13.5 to $2.6 \mathrm{~g} / \mathrm{mL}$ and from 6.8 to $5.3 \mathrm{~g} / \mathrm{mL}$, respectively. On the other hand, in the occipital pole more than $2 \mathrm{~cm}$ from the primary focus and the area of treatment, patient 2 showed progression, with an increase in maximum standardized uptake value. ${ }^{18} \mathrm{~F}$-labeled fluoroethyltyrosine control studies over 3 y gave normal results, without evidence of residual or recurrent tumor, for patient 1 , whereas the third patient had residual tumor and showed a slight increase in uptake ratios (Table 2). Patient 2 died before ${ }^{18} \mathrm{~F}$-fluoroethyltyrosine PET could be performed, and patient 3 before control ${ }^{18} \mathrm{~F}-\mathrm{FDG}$ PET could be performed. The results of the follow-up ${ }^{68} \mathrm{Ga}$-DOTATOC PET studies correlated well with the ${ }^{18} \mathrm{~F}-\mathrm{FDG}$ PET and ${ }^{18}$ F-fluoroethyltyrosine PET findings (14).

\section{DISCUSSION}

The concept of peptide receptor-mediated radiotherapy is novel in cancer treatment, and recent years have given the first results on its use in clinical oncology $(7,9,15)$. Published data on the use of ${ }^{90}$ Y-DOTATOC in patients with neuroendocrine tumors have been positive $(8)$. To our knowledge, this is the first report of the therapeutic application of this radionuclide to a group of patients with recurrent highgrade glioblastoma (WHO grade IV). According to the imaging procedures used (MRI and multimodal PET with ${ }^{68} \mathrm{Ga}$-DOTATOC, ${ }^{18} \mathrm{~F}-\mathrm{FDG}$, and ${ }^{18} \mathrm{~F}$-fluoroethyltyrosine), the patients showed a partial to complete response to therapy with ${ }^{90}$ Y-DOTATOC. Furthermore, the patients' quality of life increased substantially and even became normal in 1 patient, who had no signs of relapse for $4 \mathrm{y}$.

The hypothesis of receptor-mediated binding of the radionuclide is supported by enhanced expression of the

\section{TABLE 1. Patient Characteristics}

\begin{tabular}{|c|c|c|c|c|c|c|}
\hline Patient no. & Age $(y)$ & Sex & Histopathology & Distribution of recurrent tumor & Cumulative dose & $\begin{array}{l}\text { Mean dose to } \\
\text { tumor tissue* }\end{array}$ \\
\hline 1 & 24 & $F$ & Glioblastoma, grade IV & Circular, around cavity & 2,220 MBq (4 cycles) & $38 \mathrm{~Gy} / \mathrm{GBq}$ \\
\hline 2 & 61 & M & Glioblastoma, grade IV & Irregular, with occipital focus & $1,950 \mathrm{MBq}$ (3 cycles) & $105 \mathrm{~Gy} / \mathrm{GBq}$ \\
\hline 3 & 62 & M & Glioblastoma, grade IV & Irregular, regional, and invasive & 1,660 MBq (3 cycles) & $150 \mathrm{~Gy} / \mathrm{GBq}$ \\
\hline
\end{tabular}

${ }^{*}$ Calculated by individual dosimetry with ${ }^{111}$ In-DOTATOC. 


\section{TABLE 2. Results}

Patient

no.

1

2

3
MRI evaluation

No recurrent tumor tissue

Necrosis in target volume

Necrosis in target volume
Improvement in Karnofsky

performance score

$60 \%-100 \%$

$70 \%-80 \%$

$60 \%-80 \%$
Change in ${ }^{18} \mathrm{~F}$-FDG PET

SUVmax during therapy

$13.5-2.6 \mathrm{~g} / \mathrm{mL}$

$6.8-5.3 \mathrm{~g} / \mathrm{mL}$

$7.3 \mathrm{~g} / \mathrm{mL}^{*}$
Change in maximum

${ }^{18} \mathrm{~F}-\mathrm{FET}$ uptake relative to contralateral background

1.07-1.3

$3.2^{*}$

$2.0-2.9$

${ }^{*}$ Follow-up data not available.

$\mathrm{SUV}_{\max }=$ maximum standardized uptake value; FET = fluoroethyltyrosine

somatostatin receptor in tumor tissue, as visualized on ${ }^{68} \mathrm{Ga}-$ DOTATOC PET (6). In addition, the constant accumulation of activity in the target volume as assessed by repeated scintigraphy using ${ }^{111} \mathrm{In}$-DOTATOC is a sign of stable binding. This therapy can be regarded as safe and without major radiogenic effects to critical organs, considering that the dose to the kidneys was less than $2 \%$ and the effective dose to the whole body was less than $1 \%$ of the tumor dose as assessed by whole-body dosimetry. Our dosimetric calculations are in accordance with previous results $(9,13)$. The dose to the tumor cells by a single cycle of locally given ${ }^{90}$ Y-DOTATOC is double that of standard whole-brain radiotherapy and spares the surrounding brain tissue. Because this new therapy can safely be repeated several times, the total dose to the tumor tissue surpasses the dose of a single standard whole-brain radiotherapy session by a factor of 10 or more. Although the introduction of biodegradable carmustine wafers (Gliadel wafers; Eisai Inc.) has led to an increase in overall survival time from 11.6 to 13.9 mo (4), the major disadvantage of local chemotherapy is limited penetration into the tumor. This limitation does not apply to local injection of ${ }^{90}$ Y-DOTATOC, because after diffusion into the tissue and binding of the compound to the receptor, the $\beta$-particles reach even $5-10 \mathrm{~mm}$ beyond the point of decay. In the first patient, the recurrent tumor was well within the boundaries of the target region, whereas the other 2 patients had relapsing tumor in more distant regions of the cerebrum, as might explain the progression of disease they experienced after several months.

\section{CONCLUSION}

Although the number of patients included in our investigation was rather low, these early experiences showed that receptor-mediated radionuclide therapy by locally injected ${ }^{90}$ Y-DOTATOC is feasible and well tolerated in patients with recurrent glioblastoma IV. Local injection of ${ }^{90}$ Y-DOTATOC resulted not only in an improved quality of life but also in an objective response of single lesions on MRI and PET. This approach represents an attractive strategy for the treatment of locally recurring or progressing glioblastoma and warrants further studies.

\section{ACKNOWLEDGMENT}

We thank John Zaknun for first introducing the intracavitary method in our department and for further fruitful discussion.

\section{REFERENCES}

1. Barnholtz-Sloan JS, Sloan AE, Schwartz AG. Relative survival rates and patterns of diagnosis analyzed by time period for individuals with primary malignant brain tumor, 1973-1997. J Neurosurg. 2003;99:458-466.

2. Stupp R, Mason WP, van den Bent MJ, et al. Radiotherapy plus concomitant and adjuvant temozolomide for glioblastoma. N Engl J Med. 2005;352: 987-1006.

3. Westphal M, Hilt DC, Bortey E, et al. A phase 3 trial of local chemotherapy with biodegradable carmustine (BCNU) wafers (Gliadel wafers) in patients with primary malignant glioma. Neuro Oncol. 2003;5:79-88.

4. Virgolini I, Yang Q, Li S, et al. Cross-competition between vasoactive intestinal peptide and somatostatin for binding to tumor cell membrane receptors. Cancer Res. 1994;54:690-700.

5. Virgolini I, Raderer M, Kurtaran A, et al. Vasoactive intestinal peptide-receptor imaging for the localization of intestinal adenocarcinomas and endocrine tumors. N Engl J Med. 1994;331:1116-1121.

6. Miederer M, Seidl S, Buck A, et al. Correlation of immunohistopathological expression of somatostatin receptor 2 with standardised uptake values in ${ }^{68}$ Ga-DOTA TOC PET/CT. Eur J Nucl Med Mol Imaging. 2009;36:48-52.

7. Merlo A, Mueller-Brand J, Maecke HR. Comparing monoclonal antibodies and small peptidic hormones for local targeting of malignant gliomas. Acta Neurochir Suppl. 2003;88:83-91.

8. Bodei L, Cremonesi M, Grana C, et al. Receptor radionuclide therapy with ${ }^{90} \mathrm{Y}$-[DOTA $]^{0}-\mathrm{Tyr}^{3}$-ocreotide $\left({ }^{90} \mathrm{Y}\right.$-DOTATOC) in neuroendocrine tumors. Eur $J$ Nucl Med Mol Imaging. 2004;31:1038-1046.

9. Virgolini I, Britton K, Buscombe J, Moncayo R, Paganelli G, Riva P. In- and Y-DOTA-lanreotide: results and implications of the MAURITIUS trial. Semin Nucl Med. 2002;32:148-155.

10. Beutler D, Avoledo P, Reubi JC, et al. Three-year recurrence-free survival in a patient with recurrent medulloblastoma after resection, high-dose chemotherapy, and intrathecal yttrium-90-labeled DOTA-Tyr3-octreotide radiopeptide brachytherapy. Cancer. 2005;103:869-873.

11. Schumacher T, Hofer S, Eichhorn K, et al. Local injection of the ${ }^{90}$ Y-labeled peptidic vector DOTA-TOC to control gliomas of WHO grades II and III: an extended pilot study. Eur J Nucl Med Mol Imaging. 2002;29:486-493.

12. Hockaday DC, Shen S, Fiveash J, et al. Imaging glioma extent with ${ }^{131} \mathrm{I}-\mathrm{TM}-601$. J Nucl Med. 2005;46:580-586.

13. Ferrari M, Cremonesi M, Bartolomei M, et al. Dosimetric model for locoregional treatments of brain tumors with ${ }^{90}$ Y-conjugates: clinical application with ${ }^{90}$ Y-DOTATOC. J Nucl Med. 2006;47:105-112.

14. Pöpperl G, Götz C, Rachinger W, et al. Serial $O$ - $\left(2-\left[{ }^{18} \mathrm{~F}\right]\right.$ fluoroethyl)-L-tyrosine PET for monitoring the effects of intracavitary radioimmunotherapy in patients with malignant glioma. Eur J Nucl Med Mol Imaging. 2006;33:792-800.

15. Kwekkeboom DJ, Teunissen J, Bakker W, et al. Radiolabeled somatostatin analog ${ }^{177} \mathrm{Lu}$-DOTA-Tyr ${ }^{3}$-octreotate in patients with endocrine gastroenteropancreatic tumours. J Clin Oncol. 2005;23:2754-2762. 